\title{
Loss of spin glass behavior in ultrathin AuFe films
}

\author{
H. Fritzsche* \\ Canadian Neutron Beam Centre, Chalk River Laboratories, National Research Council Canada, \\ Bldg. 459, Chalk River, Ontario, Canada KOJ $1 \mathrm{JO}$ \\ J. M. van der Knaap and M. B. S. Hesselberth \\ Kamerlingh Onnes Laboratory, Leiden University, Leiden, The Netherlands \\ G. J. Nieuwenhuys \\ Research Department Condensed Matter Research with Neutrons and Muons, Paul-Scherrer Institute, Villigen, Switzerland \\ and Kamerlingh Onnes Laboratory, Leiden University, Leiden, The Netherlands
}

(Received 27 November 2009; revised manuscript received 10 February 2010; published 14 April 2010)

\begin{abstract}
We determined the temperature dependence of the magnetization of thin $\mathrm{Au}_{97} \mathrm{Fe}_{3}$ films in the thickness range between 1 and $50 \mathrm{~nm}$ by performing polarized neutron reflectivity measurements in a magnetic field of $6 \mathrm{~T}$ in a temperature range from 200 down to $2 \mathrm{~K}$. For the films in the thickness range from 50 to $2 \mathrm{~nm}$, we observed a Brillouin-type behavior of noninteracting Fe atoms down to $50 \mathrm{~K}$ followed by a strong reduction compared to the Brillouin curve below $50 \mathrm{~K}$. This reduction in the magnetization is a measure of the spin glass frustration and depends on the film thickness below $20 \mathrm{~nm}$ as shown in our previous study [M. Saoudi, H. Fritzsche, G. J. Nieuwenhuys, and M. B. S. Hesselberth, Phys. Rev. Lett. 100, 057204 (2008)] on $\mathrm{Au}_{97} \mathrm{Fe}_{3}$ films. The present study is a continuation of this work extending the thickness range down to $1 \mathrm{~nm}$. It shows that below $10 \mathrm{~nm}$, the reduction in the spin glass magnetization compared to a Brillouin-type behavior decreases with decreasing film thickness. Finally, the magnetization of the 1-nm-thick film could be described with a Brillouin function also below $50 \mathrm{~K}$ proving that ultrathin $\mathrm{Au}_{97} \mathrm{Fe}_{3}$ layers below $1 \mathrm{~nm}$ do not show spin glass behavior anymore but are paramagnetic.
\end{abstract}

DOI: 10.1103/PhysRevB.81.132402

PACS number(s): 75.50.Lk, 61.05.fj, 75.70.-i

Spin glass systems have been of interest to theorists as well as experimentalists for decades. ${ }^{1}$ For thin films, the focus has been on finite-size effects, ${ }^{2-8}$ the crossover from twoto three-dimensional behavior, ${ }^{9}$ and the existence of a surface anisotropy. ${ }^{8,10-14}$ The challenge from an experimental point of view is to provide reliable experimental data on the magnetization of single films in the nanometer range containing only a few atomic percent of magnetic atoms in a nonmagnetic matrix.

Superconducting quantum interference device magnetometry has been successfully employed to study bulk properties ${ }^{15,16}$ but is not adequate to measure the magnetization of spin glass films in the nanometer range because of the small magnetic signal compared to the huge diamagnetic background of the substrate. Therefore, magnetometry experiments on thin spin glass films reported in literature were all performed on multilayers in order to increase the signal. ${ }^{2,5}$ However, it is always questionable whether multilayer data represent the true single-film properties because of structural differences as the film gets thicker and an uncomplete decoupling of the individual spin glass layers. ${ }^{17}$

Finite-size effects were also inferred from the vanishing of a cusp in anomalous Hall-effect measurements ${ }^{3}$ of $\mathrm{AuFe}$ thin films at about $12 \mathrm{~nm}$. However, the vanishing of a cusp does not necessarily mean a vanishing of the spin glass frustration. Susceptibility measurements showed that the cusp in $\mathrm{AuFe}$ samples is smeared out in magnetic fields ${ }^{15}$ and anomalous Hall-effect measurements showed that the cusp vanishes in large magnetic fields. ${ }^{3,18}$ Using polarized neutron reflectometry (PNR), we were able to show ${ }^{7,19}$ that thin AuFe films show spin glass behavior in a large magnetic field of
$6 \mathrm{~T}$. Therefore, one has to be cautious when drawing conclusions from the vanishing of a cusp in either susceptibility or Hall-effect measurements.

PNR has the advantage to measure the magnetization directly and the contribution of the substrate to the measured signal does not obscure the tiny signal of the thin film as has been shown, e.g., in ultrathin Fe films. ${ }^{20,21}$ We have successfully employed the technique of PNR to study the properties of thin AuFe spin glass films. ${ }^{7,19,22}$ Recently we observed a finite-size effect in the temperature dependence of the magnetization of single $\mathrm{Au}_{0.97} \mathrm{Fe}_{0.03}$ films. ${ }^{7}$ The films in the range from 500 to $20 \mathrm{~nm}$ showed a Brillouin-type behavior from $295 \mathrm{~K}$ down to $50 \mathrm{~K}$ with a constant magnetization of $0.9 \mu_{B}$ per Fe atom below $30 \mathrm{~K}$ whereas the magnetization of the 10-nm-thick film could be fitted with a Brillouin function down to $20 \mathrm{~K}$ followed by a constant magnetization of $1.3 \mu_{B}$. So, the temperature dependence of the magnetization in AuFe films deviates from bulk behavior below $20 \mathrm{~nm}$ but it still shows a typical spin glass behavior at $10 \mathrm{~nm}$. This is in agreement with recent low-energy muon-spin-rotation experiments $^{8}$ on $\mathrm{AuFe}$ films, where Morenzoni et al. found a reduced freezing in a 10 -nm-thick region below the AuFe film surface.

The present PNR study is a continuation of this previous investigation ${ }^{7}$ by extending the film thickness range from 10 $\mathrm{nm}$ further down to $1 \mathrm{~nm}$ and applying the same experimental conditions as before. We prepared AuFe alloy films with an Fe concentration of 3 at. \% and thicknesses of $t=1,2,5$, 10 , and $50 \mathrm{~nm}$ by cosputtering high-purity $\mathrm{Au}$ and $\mathrm{Fe}$ onto a 2.6-nm-thick $\mathrm{Mo}_{0.7} \mathrm{Ge}_{0.3}$ buffer layer on a silicon substrate. With this $\mathrm{Mo}_{0.7} \mathrm{Ge}_{0.3}$ buffer layer, an island growth could be 
avoided. At the same time, the $\mathrm{Mo}_{0.7} \mathrm{Ge}_{0.3}$ layer acts as a diffusion barrier to avoid an interdiffusion of the AuFe film with the Si. Previous PNR experiments ${ }^{7,19}$ were performed on AuFe samples that did not have this $\mathrm{Mo}_{0.7} \mathrm{Ge}_{0.3}$ buffer layer. The films were analyzed using Rutherford backscattering and electron microprobe analysis showing that the thickness and composition were homogeneous. The samples were cooled down in a magnetic field of $6 \mathrm{~T}$ to avoid time effects of the magnetization which occur when measuring the magnetization after a zero-field cooling. ${ }^{23,24}$

PNR experiments were performed on the C5 spectrometer at the neutron research reactor NRU in Chalk River, Canada. We used a $\mathrm{Cu}_{2} \mathrm{MnAl}$ Heusler crystal at $\lambda=0.237 \mathrm{~nm}$ as monochromator and analyzer along with a PG filter ${ }^{25}$ to reduce the higher-order contamination $(\lambda / 2, \lambda / 3)$. The magnetic field was provided by a cryomagnet with the magnetic field being in the sample plane and perpendicular to the scattering plane. In this setup, ${ }^{26}$ we achieved a $96 \%$ polarization of the incoming neutron beam, as determined from the measured flipping ratio of 26. In the used specular reflection geometry, the reflected angle $\theta_{f}$ is always equal to the incident angle $\theta_{i}\left(\theta=\theta_{i}=\theta_{f}\right)$. The scattering vector $\mathbf{q}=\mathbf{k}_{\mathbf{f}}-\mathbf{k}_{\mathbf{i}}$ is perpendicular to the film surface with $k_{f}$ and $k_{i}$ being the wave vector of the reflected and incoming beam. For the case that the sample's magnetization is parallel to the external field, the interaction of neutrons with a film in a reflectometry experiment can be described by the Fermi pseudopotential $V^{ \pm}$,

$$
V^{ \pm}=\frac{2 \pi \hbar^{2}}{m} \rho\left(b_{n} \pm b_{m}\right),
$$

where $m$ denotes the neutron mass, $\rho$ the atomic density, $b_{n}$ the nuclear scattering length, and $b_{m}$ the magnetic scattering length. The superscripts + and - indicate that the scattering potential is different for neutrons with their spins aligned parallel (+, up neutrons) or antiparallel ( - , down neutrons) to the external field. The nuclear scattering length depends on the elements and their isotopes in the sample, ${ }^{27}$ the magnetic scattering length is directly proportional to the magnetization of the sample with $b_{m}=c \mu$, where $c=2.695 \mathrm{fm} /$ bohr magneton is a conversion constant and $\mu$ is the magnetic moment per atom. For the spin-up neutrons, the magnetic potential adds to the nuclear one whereas for the spin-down neutrons, the magnetic potential is subtracted from the nuclear potential. This leads to different reflectivity curves for the two spin states what is used to determine the magnetic moment of magnetic films. The quantities $\rho b_{n}$ and $\rho b_{m}$ are the nuclear and magnetic scattering length density (SLD), respectively.

Figure 1 displays the polarized neutron reflectivities $R^{+}(+)$and $R^{-}$(open circles) of the 1-nm-thick $\mathrm{Au}_{97} \mathrm{Fe}_{3}$ film as a function of the scattering vector $q$, measured at $5 \mathrm{~K}$ in a magnetic field of $6 \mathrm{~T}$. In our case, $R^{+}$and $R^{-}$are nearly identical because the magnetization of the AuFe film is extremely small. Therefore, it is better to plot the asymmetry $A$ defined as

$$
A=\left(R^{+}-R^{-}\right) /\left(R^{+}+R^{-}\right)
$$

as a function of $q$ in order to visualize the magnetic properties of the film.

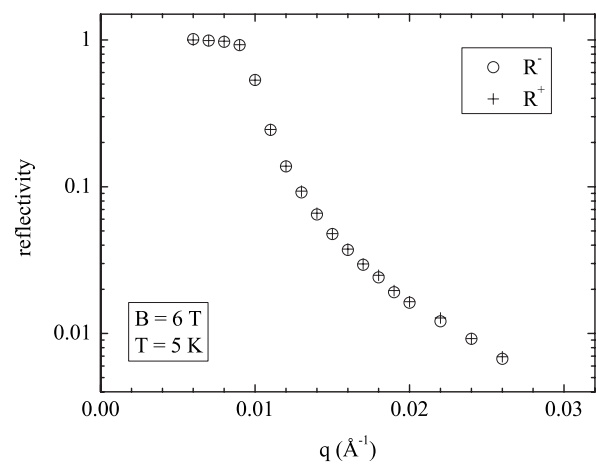

FIG. 1. Measured neutron reflectivities $R^{+}(+)$and $R^{-}$(open circles) as a function of the scattering vector $q$ for a 1-nm-thick $\mathrm{Au}_{97} \mathrm{Fe}_{3}$ film at a temperature $T=5 \mathrm{~K}$ in an external magnetic field of $6 \mathrm{~T}$.

Figure 2 shows the asymmetry for the 1-nm-thick $\mathrm{Au}_{97} \mathrm{Fe}_{3}$ film as calculated from the reflectivity data shown in Fig. 1 using Eq. (2). The solid line represents a fit that was obtained by fitting simultaneously the spin-up and spin-down reflectivity using Parratt's recursion formalism. ${ }^{28}$ We used a twolayer model that comprises of a $\mathrm{Au}_{97} \mathrm{Fe}_{3}$ film on a $\mathrm{Mo}_{0.7} \mathrm{Ge}_{0.3}$ layer on top of a silicon substrate. The result for the 1-nmthick $\mathrm{Au}_{97} \mathrm{Fe}_{3}$ film at $5 \mathrm{~K}$ is a magnetic SLD of 0.17 $\times 10^{-6} \AA^{-2}$ and a nuclear SLD of $4.4 \times 10^{-6} \AA^{-2}$. The magnetic SLD was converted into an average magnetic moment $\bar{\mu}$ along the magnetic field of $3.6 \mu_{B}$ per Fe atom by assuming the same number density for the $\mathrm{Fe}$ as for the $\mathrm{Au}$ atoms. ${ }^{22}$ For comparison, there is a dashed line in Fig. 2 representing the simulated asymmetry for a magnetic moment of $0.9 \mu_{B}$ per $\mathrm{Fe}$ atom, the value measured earlier ${ }^{7}$ below $30 \mathrm{~K}$ for films thicker than $20 \mathrm{~nm}$. Despite the fact that the error bars are quite large, it is obvious that the magnetization of the 1 -nm-thick film is much larger than $0.9 \mu_{B}$ per Fe atom.

In order to see whether the 1-nm-thick $\mathrm{Au}_{97} \mathrm{Fe}_{3}$ film still shows spin glass behavior, we compared the measured temperature-dependent spin glass magnetization with the magnetization of noninteracting $\mathrm{Fe}$ atoms with $\mu=4 \mu_{B}$ per Fe atom, described by the Brillouin function. This is shown in Fig. 3, where the solid circles correspond to the values for

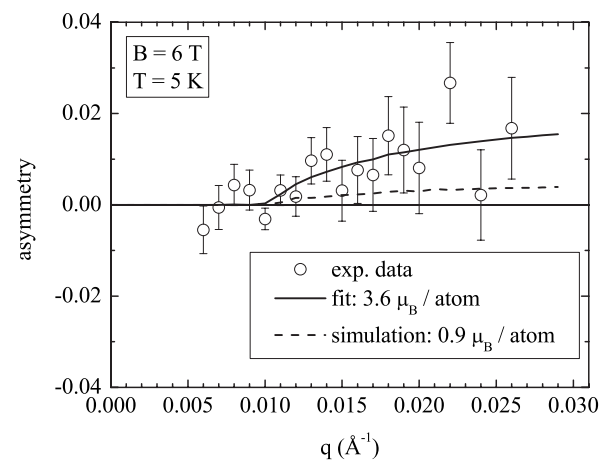

FIG. 2. Asymmetry as a function of the scattering vector $q$ as calculated from the reflectivities of a 1-nm-thick $\mathrm{Au}_{97} \mathrm{Fe}_{3}$ film shown in Fig. 1, measured at $5 \mathrm{~K}$ in a field of $6 \mathrm{~T}$. The solid line represents a fit to the data and the dashed line corresponds to a simulation with a magnetization of $0.9 \mu_{B}$ per $\mathrm{Fe}$ atom. 


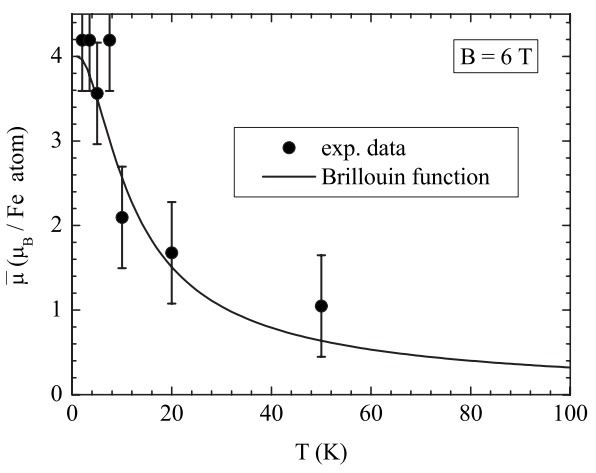

FIG. 3. Averaged magnetic moment per Fe atom versus temperature of a 1-nm-thick $\mathrm{Au}_{97} \mathrm{Fe}_{3}$ films as derived from the fit to the PNR data (solid circles) compared to a Brillouin function with $J$ $=2$ for isolated $\mathrm{Fe}$ atoms (solid line).

the average magnetic moment $\bar{\mu}$ as derived from fits to the reflectivity curves. The solid line is the average magnetic moment of noninteracting $\mathrm{Fe}$ atoms calculated as the product of $\mu=4 \mu_{B}$ and the Brillouin function $B(J, x)$, where $J=2$ is the total angular momentum and $x=\mu B / k_{B} T$ with $B$ the applied magnetic field, $k_{B}$ the Boltzmann constant, and $T$ the temperature. Within the errors, the thin-film data can be well described with the Brillouin function. This means that the $1 \mathrm{~nm}$ film does not show a spin glass behavior anymore but behaves like a paramagnetic film.

For all the other film thicknesses measured in this study, we observed a distinct reduction in the film magnetization compared to the Brillouin function as can be seen in Fig. 4, where the solid circles are the experimental data and the solid lines represent the calculated Brillouin curve for noninteracting $\mathrm{Fe}$ atoms. The average magnetic moment measured at $2 \mathrm{~K}$ increases from $0.9 \mu_{B}$ per Fe atom for the 10nm-thick film to $1.3 \mu_{B}$ for the $5 \mathrm{~nm}$ film to $2.5 \mu_{B}$ for the 2 $\mathrm{nm}$ film, and finally to $4.2 \mu_{B}$ for the $1 \mathrm{~nm}$ film. This nicely shows the transition from a spin glass with bulk behavior at a film thickness of $50 \mathrm{~nm}$ to a paramagnetic film at $1 \mathrm{~nm}$.

In conclusion, we have performed PNR measurements on thin $\mathrm{Au}_{97} \mathrm{Fe}_{3}$ single films in a field of $6 \mathrm{~T}$ and successfully determined the magnetization as a function of temperature. The magnetization of the $\mathrm{Au}_{97} \mathrm{Fe}_{3}$ films can be described with a Brillouin function down to a temperature of about $50 \mathrm{~K}$, followed by a strong reduction compared to the Bril-

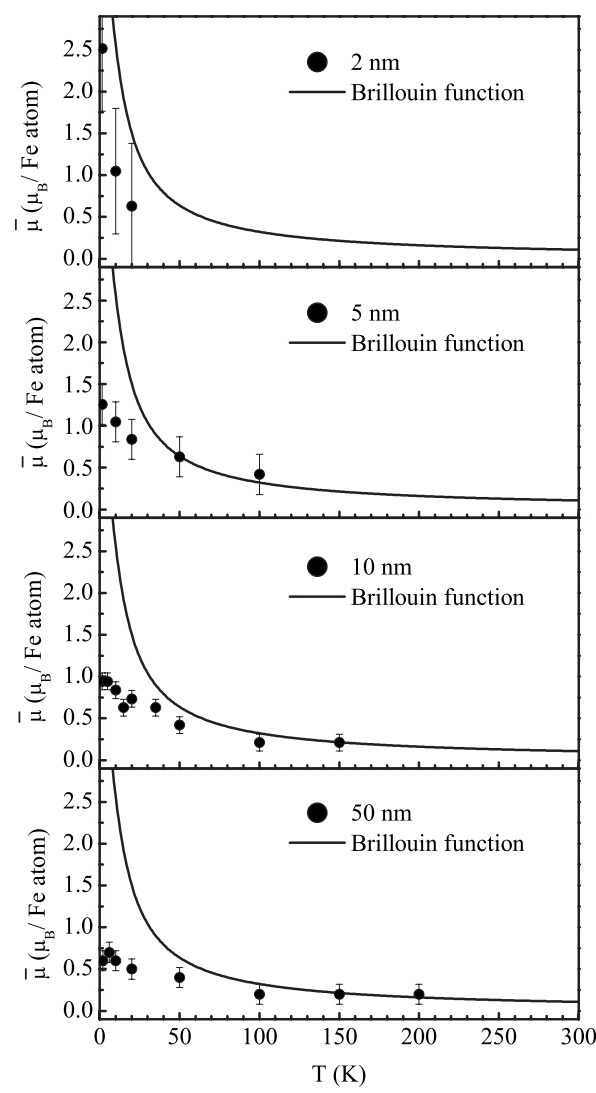

FIG. 4. Averaged magnetic moment per Fe atom versus temperature of $\mathrm{Au}_{97} \mathrm{Fe}_{3}$ films as derived from the fit to the PNR data (solid circles) compared to a Brillouin function with $J=2$ for isolated $\mathrm{Fe}$ atoms (solid line).

louin curve for films with a thickness from 50 to $2 \mathrm{~nm}$ in agreement with previous PNR experiments on thin $\mathrm{Au}_{97} \mathrm{Fe}_{3}$ films. ${ }^{7}$ The only exception is the 1-nm-thick film that can be fitted with the Brillouin curve even for temperatures down to $2 \mathrm{~K}$. Our data clearly prove a phase transition to a spin glass state in large magnetic fields down to $2 \mathrm{~nm}$ film thickness and a paramagnetic behavior for the 1-nm-thick $\mathrm{Au}_{97} \mathrm{Fe}_{3}$ film. Furthermore, the magnetization of the $\mathrm{Au}_{97} \mathrm{Fe}_{3}$ films in the thickness range between 10 and $2 \mathrm{~nm}$ show a clear size dependence.

\footnotetext{
*helmut.fritzsche@nrc.gc.ca

${ }^{1}$ K. Binder and A. P. Young, Rev. Mod. Phys. 58, 801 (1986).

${ }^{2}$ G. G. Kenning, J. M. Slaughter, and J. A. Cowen, Phys. Rev. Lett. 59, 2596 (1987).

${ }^{3}$ H. Vloeberghs, J. Vranken, C. Van Haesendonck, and Y. Bruynseraede, Europhys. Lett. 12, 557 (1990).

${ }^{4}$ G. Neuttiens, J. Eom, C. Strunk, V. Chandrasekhar, C. Van Haesendonck, and Y. Bruynseraede, Europhys. Lett. 34, 617 (1996).

${ }^{5}$ G. G. Kenning, J. Bass, W. P. Pratt, Jr., D. Leslie-Pelecky, L. Hoines, W. Leach, M. L. Wilson, R. Stubi, and J. A. Cowen, Phys. Rev. B 42, 2393 (1990).
}

${ }^{6}$ L. Hoines, R. Stubi, R. Loloee, J. A. Cowen, and J. Bass, Phys. Rev. Lett. 66, 1224 (1991).

${ }^{7}$ M. Saoudi, H. Fritzsche, G. J. Nieuwenhuys, and M. B. S. Hesselberth, Phys. Rev. Lett. 100, 057204 (2008).

${ }^{8}$ E. Morenzoni, H. Luetkens, T. Prokscha, A. Suter, S. Vongtragool, F. Galli, M. B. S. Hesselberth, N. Garifianov, and R. Khasanov, Phys. Rev. Lett. 100, 147205 (2008).

${ }^{9}$ D. S. Fisher and D. A. Huse, Phys. Rev. B 36, 8937 (1987).

${ }^{10}$ O. Újsághy, A. Zawadowski, and B. L. Gyorffy, Phys. Rev. Lett. 76, 2378 (1996).

${ }^{11}$ O. Újsághy and A. Zawadowski, Phys. Rev. B 57, 11598 (1998). 
${ }^{12}$ O. Újsághy, L. Szunyogh, and A. Zawadowski, Phys. Rev. B 75, 064425 (2007).

${ }^{13}$ E. Seynaeve, K. Temst, F. G. Aliev, C. Van Haesendonck, V. N. Gladilin, V. M. Fomin, and J. T. Devreese, Phys. Rev. Lett. 85, 2593 (2000).

${ }^{14}$ V. N. Gladilin, V. M. Fomin, and J. T. Devreese, Physica B 294-295, 302 (2001).

${ }^{15}$ V. Cannella and J. A. Mydosh, Phys. Rev. B 6, 4220 (1972).

${ }^{16}$ L. Lundgren, P. Svedlindh, and O. Beckman, Phys. Rev. B 26, 3990 (1982).

${ }^{17}$ R. Stubi, L. Hoines, R. Loloee, I. Kraus, J. A. Cowen, and J. Bass, Europhys. Lett. 19, 235 (1992).

${ }^{18}$ S. P. McAlister and C. M. Hurd, Solid State Commun. 19, 881 (1976).

${ }^{19}$ H. Fritzsche, M. Saoudi, K. Temst, and C. Van Haesendonck, Physica B 397, 47 (2007).
${ }^{20}$ J. A. C. Bland, C. Daboo, B. Heinrich, Z. Celinski, and R. D. Bateson, Phys. Rev. B 51, 258 (1995).

${ }^{21}$ T. Nawrath, H. Fritzsche, F. Klose, J. Nowikow, and H. Maletta, Phys. Rev. B 60, 9525 (1999).

${ }^{22}$ J. Swerts, K. Temst, C. Van Haesendonck, H. Fritzsche, V. N. Gladilin, V. M. Fomin, and J. T. Devreese, Europhys. Lett. 68, 282 (2004).

${ }^{23}$ C. N. Guy, J. Phys. F: Met. Phys. 5, L242 (1975).

${ }^{24}$ R. V. Chamberlin, M. Hardiman, L. A. Turkevich, and R. Orbach, Phys. Rev. B 25, 6720 (1982).

${ }^{25}$ J. Bergsma and C. van Dijk, Nucl. Instrum. Methods 51, 121 (1967).

${ }^{26}$ H. Fritzsche, Rev. Sci. Instrum. 76, 115104 (2005).

${ }^{27}$ V. F. Sears, Neutron News 3, 26 (1992).

${ }^{28}$ L. G. Parratt, Phys. Rev. 95, 359 (1954). 